\title{
Reflexiones en torno al currículo educativo y la perspectiva de género*
}

\section{Reflections on the educational curriculum and the gender perspective}

María del Carmen Jiménez Ruiz ${ }^{\star *}$ Adriana González Perdomo***
Recibido: 13-mar-2019

Aceptado: 13-nov-2019

\section{Resumen}

El presente artículo, de corte teórico-reflexivo, procura dar respuesta al interrogante sobre el papel de la educación superior en el incremento de experiencias de divergencia entre los géneros; se pretende exponer las ideas elaboradas por las autoras a partir de una minuciosa revisión bibliográfica y reflexiones personales.

Se plantea la importancia de realizar raciocinios en torno al currículo como agente dinamizador en los procesos del saber pedagógico y la enseñanza, los cuales pueden causar rupturas entre el conocimiento y el ejercicio docente. El razonamiento a través de la interpelación sobre el diseño curricular, los contenidos programáticos, las experiencias educativas y las interacciones entre los sujetos que transitan los desarrollos curriculares, fundan en Unicatólica una búsqueda del reconocimiento a la igualdad de género que contribuya a la construcción democratizadora de la sociedad.

Se explora cómo se ha abordado el tema del currículo desde una perspectiva histórica e interpela la formación de actitudes, surge así la importancia de incorporar las prácticas educativas, procesos curriculares y perspectiva de género en pos de sociedades incluyentes y para servir como agentes de procesos de corresponsabilidad entre los géneros.
Para concluir, se puede afirmar que es viable realizar un análisis del vínculo género-educación superior, para conseguirlo resulta ineludible desplegar habilidades y acciones conjuntas, desde la perspectiva de genero.

\section{Palabras clave}

currículo; formación en valores; perspectiva de género.

\section{Abstract}

The present article, of a theoretical-reflective nature, tries to answer the question about the role of higher education in increasing experiences of divergence between genders. The aim is to expose the ideas elaborated by the authors from a meticulous bibliographic review and personal reflections.

The importance of making reasonings around the curriculum as a dynamic agent in the processes of pedagogical knowledge and teaching, which can cause breaks between knowledge and teaching practice is raised. The paper studies the reasoning through the interpellation on the curricular design, the programmatic contents, educational experiences and the interactions between the subjects that transit the curricular developments, found in Unicatólica a search for the recognition of

\footnotetext{
* Este artículo teórico-reflexivo hace parte de las deliberaciones presentadas al interior del proyecto, denominado "Los procesos curriculares y la incorporación de la perspectiva de género en la Fundación Universitaria Católica Lumen Gentium" del Municipio de Santiago de Cali (Valle del Cauca). ** María del Carmen Jiménez, Programa de Psicología, Fundación Universitaria Católica Lumen Gentium, Unicatólica. Psicóloga. Universidad del Valle. Especialista en investigación en contextos de docencia universitaria y magíster en Educación, Universidad de San Buenaventura. ORCID: 000-001-9949-1484

*** Adriana González Perdomo, Socióloga, Universidad del Valle. Perfeccionamiento, Universidad Nacional de Colombia sede Bogotá y Universitá Internazionale delle Istituzioni dei popoli per la pace.
} 
gender equality that contributes to democratizing the construction of society.

It explores how the subject of the curriculum has been approached from a historical perspective and questions the formation of attitudes, thus the importance of incorporating educational practices, curricular processes and gender perspective in pursuit of inclusive societies and to serve as agents of processes of co-responsibility between genders.

To conclude, it can be affirmed that it is feasible to carry out an analysis of the gender-higher education link. To achieve this, it is unavoidable to deploy joint skills and actions, from the gender perspective.

\section{Keywords} perspective.

curriculum; training in values; gender

\section{Introducción}

Deliberar sobre el currículo lleva a pensar la manera en que este permea lo institucional, familiar y comunitario, por medio de estrategias que movilizan procesos de formación en los cuales la educación se convierte en un acto, esto significa según Grundy que "si el currículo es una práctica, quiere decir que todos los que participan en ella son sujetos, no objetos; es decir, elementos activos" (citado por López y Luna, 2011, p. 3). En él se incorporan planes didácticos y la mejora de los afectos, en procura de búsquedas más humanizantes que cuantitativas, que afronten los desafíos cambiantes inmersos en los procesos de enseñanza-aprendizaje, bajo realidades históricas y culturales, e incentivar así a estar abiertos a la cuestión crítica y poder transportar esto a la práctica.

Según Casarini (2010), la finalidad del currículo es desarrollar la personalidad, aptitudes y capacidades; adquirir aprendizajes para entender el mundo, actuar en él y comprenderlo; adquirir saberes coherentes e interdisciplinares, convertirse en una organización flexible de la enseñanza; atender a la diversidad.

\section{Como lo enuncian Carreras et al. (1996),}

[...] los fines en la educación no deben limitarse a instruir y a desarrollar el conocimiento, sino que también deben contribuir al desarrollo de la persona de forma global y de la sociedad. La necesidad de una sociedad en la que las relaciones interpersonales estén basadas en el respeto y la tolerancia, y la participación social crítica y constructiva son razones suficientes que justifican la integración de valores y actitudes en el currículo escolar, donde los contenidos generales sean: conceptuales, procedimentales y actitudinales. (p. 25)

\section{Breve estado de la cuestión sobre el currículo}

En el desarrollo histórico se expone un currículo integrado con la formación en valores. Se observa en la historia del pensamiento educativo, especialmente en la sociedad industrial, que Durkheim (Francia) y Dewey (Estados Unidos) fueron quienes enfatizaron en el valor de una educación centrada en los procesos de ciudadanía, desarrollo industrial y progreso. Dewey (1952) asignó a la escuela el concepto de moralidad como conducta responsable, ligada a la democracia. Concluyó que "la educación no puede considerarse instrumento democratizador, sin afirmarse antes en la democracia" (p. 109). La democracia -para este autor- está impregnada por la educación para la libertad y el progreso, involucra intrínsecamente tres aspectos: democracia, libertad y progreso (individual y social). Concluye Dewey que tanto en la construcción de los medios educativos (currículo), como en la valoración de sus consecuencias, el profesor se convertiría en un artista, porque la misma crítica, en su más alta acepción, es un arte (Sacristán y Pérez, 1985, p. 8). 
Para Durkheim (1976), las sociedades requieren un límite que regule y ordene la actividad del hombre, puesto que "la disciplina escolar debe crear en el alumno esta necesidad de autolimitación". Mientras que Magendzo (2008) considera que el currículum tiene como propósito deliberado y manifiesto la formación y el desarrollo en los estudiantes, de una identidad, una conciencia, una moral que les permita definirse como personas individuales que se asumen a sí mismos, es sin duda alguna, muy trascendente. Stenhouse, citado por Gallego (2012), lo reafirma al considerar que "el currículo es un instrumento potente e inmediato para la trasformación de la enseñanza”, y por supuesto, para el aprendizaje.

Como lo plantea De la Herrán (2012), un 'currículo' se puede referir a un periodo, cultura, pueblo, escuela de pensamiento, etc. Por otro lado, en cada época o lugar un currículo puede desarrollar formas diversas. Por ejemplo, humanísticas, artísticas, académicas, deportivas, etc. Además, puede ser impuesto, propuesto o espontáneo. Continúa el autor:

Por lo tanto es de manifiesto que la definición de currículo no va a ser universal, inmutable y definitiva; siempre va a estar supeditado a dinámicas de cambio que lo enriquecen y lo adaptan a las necesidades educativas y sociales. Es por esta razón que el currículo debe estar constantemente expuesto a evaluaciones e investigaciones que lo permitan reevaluar y transformar a favor de los procesos de formación. (De la Herrán, 2012, p. 290)

La intencionalidad del currículo inmerso en un sistema educativo pone en contacto procesos relacionados con la enseñanza y el aprendizaje, para vislumbrar nuevas miradas de desarrollo humano que potencialicen la educación, la cual en el nuevo siglo constituye para Pérez (2008), un vehículo socializador y mediador de los seres humanos, el conocimiento y los ámbitos de interacción geográficos, históricos, sociales y culturales.
Sin embargo, existen frente a él diversas definiciones, las cuales involucran formas de acercamiento que varían: los objetivos de la educación, las transformaciones en las situaciones socioculturales, las vivencias de los estudiantes, las prácticas educativas, los contenidos, la baja calidad académica, los déficits en formación pedagógica y científica de los docentes, reflexiones en torno al proceso de desarrollo del currículo.

Las investigaciones con enfoque de género involucran ejecutar un examen claro de las correspondencias entre los géneros, analizar los componentes que transgreden ese absolutismo; los establecimientos educativos que castigan o certifican los cánones, los compromisos y los términos de género; así como la apreciación de las mujeres y los hombres como individuos pertenecientes a una sociedad, delimitados por un acervo de relaciones, restringidos socialmente y como resultado de una humanidad concreta.

\section{Orientaciones teóricas relacionadas con el sexismo en la educación}

La expresión sexismo surgió en los Estados Unidos en la década de 1970 por afinidad con la expresión discriminación étnica, con la única intención de manifestar que el sexo para las mujeres proporciona una fuente de discriminación, subordinación y desvalorización. Esta expresión la utilizaron los grupos feministas que en aquel momento estaban surgiendo.

Con la indemnidad de la discriminación, el género femenino sale mal librado por las constantes agresiones a las que están expuestas, amenazas indirectas que son consecuencia del régimen político y social del Estado. Todo lo anteriormente expuesto dio como resultado que las mujeres, a consecuencia del género, desafiaran situaciones que les obstaculizaba participar con integridad en las organizaciones en las que viven. 
La discriminación se expone en el ámbito de la academia a través del currículo manifiesto y del currículo oculto. En el currículo manifiesto o abierto, esta se expresa en los manuales, libros, artículos, textos, material pedagógico, acontecimientos, entre otros, en los cuales sobresalen las diligencias ejecutadas por el género masculino, mientras se minimiza, prescinde o invisibiliza tanto a niñas como a mujeres.

En el currículo oculto la discriminación sexual se exterioriza a través de la forma de trato particular a mujeres y hombres a través del lenguaje, los aspavientos, la expresión de la voz, la periodicidad y la permanencia de la atención compensada a unas y otros, el tipo de cuestionamientos y de contestaciones, la broma, la parodia y el sarcasmo al estudiantado por parte de los profesores.

Dentro de cada cultura se establece un conjunto de destrezas, doctrinas, disertaciones y alegorías sociales que imputan características definidas tanto a mujeres como a hombres. Dicho fundamento simbólico, que en los dogmas sociales se designa como género, normaliza y determina el comportamiento objetivo y subjetivo de los individuos, de tal manera que, a través del procedimiento de configuración del género, la sociedad cimienta las doctrinas de lo que se presume como "oportuno", "inseparable", "esencial" y/o "particular" de cada género.

Prevalecen componentes del conocimiento que fortalecen los mecanismos que van a perpetuar el sometimiento de la mujer, por lo tanto, se hace ineludible y necesario continuar con la denuncia de las expresiones discriminatorias que aún se observan en pleno siglo XXI dentro de las instituciones educativas, así como los alcances injustos e improcedentes que ello provoca. Las discrepancias entre los géneros no se pueden transformar si no se tienen en cuenta los importes de la sociedad que han imposibilitado la igualdad, de manera especial, los resultados que han generado el fraccionamiento del ambiente privado equivalente a la mujer y el ambiente público relacionado con el hombre.

Las indagaciones sobre la discriminación en las instituciones educativas tienen su inicio a mediados de los años ochenta con la sociología de la educación que anteriormente no recibía la atención necesaria en este ámbito. En primer lugar, porque no se veía a la escuela como un ente que discriminara a sus estudiantes, por el contrario, esta se consideraba la principal forma de certificar la paridad de congruencias, porque de alguna u otra manera, posibilitaba que el estudiante lograra un nivel de formación educativa definido para acceder a trabajos justamente gratificados. La indagación sociológica fue la encargada de demostrar que la institución educativa, por una parte, no favorecía a la igualdad de situaciones, y por otra, representaba las discrepancias entre los diferentes grupos sociales.

Por lo tanto, a partir de este tiempo y con la formulación del concepto de coeducación, se desplegó un nuevo horizonte didáctico coherente con el ideal de una educación igualitaria para los dos géneros, el cual se cercena con el proceso de asimilación segregacionista de género perpetrada en la institución educativa, cuyo objetivo final es que se presenten en el aula de clases, patrones culturales de igualdad, tanto para niños como para niñas y se genere una verdadera integración.

Hay registros sobre cómo el inicio del currículo en Colombia contempla lo enunciado desde la cultura anglosajona, fundamentada directamente en el plan de estudios. Fue en 1971 cuando el profesor Márquez, funcionario de la Universidad Nacional, consideró que el currículo hacía parte del lenguaje educativo, lo involucró con "acciones que realiza el estudiante tutelado por el profesor para así influir en su conducta y aprendizaje, las consecuencias a la estructura curricular, afectan al individuo como al contexto [en] que interactúa" (Fernández, 2016). 
La manera en que se despliega el currículo se encuentra en Fernández (2008), quien expone que durante 1974 y 1975, el Ministerio de Educación Nacional (MEN) consideró -después de varios debates- desarrollar el Plan de Mejoramiento Cualitativo bajo los lineamientos de los siguientes tres aspectos: diseño curricular, capacitación de docentes y distribución de materiales.

A nivel de política educativa y administrativa en Colombia, el currículo discurre en cuatro momentos: 1) usos del término currículo en Colombia; 2) institucional; 3) político administrativo; y 4) fenómeno académico. En los siguientes párrafos se exponen las reformas expedidas en actos administrativos que establecen el currículo, Decreto 088 de 1976, según el cual se nacionaliza la educación primaria y secundaria.

Mediante el Decreto 1816 de 1978 se crearon los Centros Experimentales Pilotos (CEP). El mejoramiento cualitativo de la educación a nivel prescolar, básica (primaria y secundaria), media vocacional e intermedia profesional se fundó en el Decreto 1419 de julio de 1978. Posteriormente, con el Decreto 181 de 1982 se constituyó el mapa educativo. Actualmente en Colombia, desde una perspectiva reglamentaria, el currículo se adhiere a un marco legal concreto señalado en la Ley General de Educación (115 de 1994), el Decreto 1860 de 1994, la Resolución 2343 de 1996, el Decreto 1290 del 2009, los lineamientos curriculares de las diferentes áreas y los estándares básicos de competencias en diferentes áreas. Esto según lo definido por la Ley 115 de 1994, en su artículo 76, que enuncia una conceptualización del currículo, planteado de la siguiente manera:

[...] el conjunto de criterios, planes de estudio, programas, metodologías, y procesos que contribuyen a la formación integral y a la construcción de la identidad cultural nacional, regional y local, incluyendo también los recursos humanos, académicos y físicos para poner en práctica las políticas y llevar a cabo el proyecto educativo institucional. (MEN, 2004)

Finalmente, para el siglo XXI, el currículo adoptó un concepto dinámico que se relaciona con el contexto y el papel transformador del docente en los estudiantes al ofrecerles una formación integral (La Francesco y Giovanni, 2003). En este sentido, el currículo está asociado a la planeación, organización e integración de actores involucrados en procesos de construcción del conocimiento, en los que se configuran aspectos relacionados con formas de potenciar el desarrollo humano a través de la educación, basados en la premisa de que los estudiantes deben aprender y los docentes deben enseñar. Es así que uno de los principios fundamentales propuestos por las Naciones Unidas en la Agenda 2030 para el Desarrollo Sostenible fue la educación, definida como:

[...] un derecho fundamental y habilitador. Para lograr este derecho, los países deben garantizar el acceso en condiciones de igualdad a una educación y un aprendizaje inclusivos, equitativos y de calidad, sin dejar a nadie atrás. La educación ha de aspirar a la plena realización de la personalidad humana y promover el entendimiento mutuo, la tolerancia, la concordia y la paz. (Organización de las Naciones Unidas para la Educación, la Ciencia y la Cultura [Unesco], 2016, p. 8)

\section{Currículo educativo y perspectiva de género}

Analizar la integración desde lo académico y la perspectiva de género permite identificar las situaciones de hombres y mujeres en sus contextos escolares, con lo que se esboza una realidad inmersa en el ambiente educativo, que de esta manera impulsa un reconocimiento de género que se potenciaría en el campo profesional.

En la actualidad ya no se puede considerar la igualdad como una analogía ni como una exigencia de un arquetipo único, por meritorio que 
este sea en términos de valoración social. Se requiere que forme parte del sistema educativo de manera intrínseca -y por consiguiente del sistema sociocultural preponderante-; una observación de la equivalencia de los contrastes socioculturales originarios de grupos humanos que no han tenido una perspectiva absoluta, pero que, no obstante, han producido a través del tiempo, hábitos, actitudes y valores imprescindibles para la vida social. Desconocer dichos aportes no es solamente proceder de forma arbitraria con respecto a los integrantes de dichos grupos, y continuar excluyéndolos en el ámbito educativo; es, asimismo, y de manera mucho más comprometedora, exceptuar de facultades y actitudes imprescindibles actualmente para nuestra conservación y por ende, para nuestra felicidad.

La exigencia a las entidades universitarias a que se pregunten acerca de la manera de llevar a cabo su actividad y dar una respuesta de manera apropiada a los requerimientos y solicitaciones socioculturales que se administran en el régimen de educación superior en el presente es necesaria e ineludible. El punto de partida es el supuesto de que los docentes se conviertan en un conjunto de protagonistas excepcionales para suscitar el cambio hacia un futuro de mayor calidad y equidad. En este apartado, la discrepancia entre mujeres y hombres se manifiesta en el permanente desarrollo de singularidad que considera la diligencia académica y manifiesta tensiones en las nociones y prácticas sobre los que se cimienta el diario quehacer académico.

A partir de la perspectiva de género, esto se percibe en la preponderancia del hombre en la creación del conocimiento inscrita al desempeño científico, la administración y misión de los establecimientos universitarios y la producción y reproducción de la estructura patriarcal en el sistema de educación superior que distribuye -de manera diferencial- a académicos según sus disciplinas, jerarquías y funciones.
Todos los procedimientos en la educación tienen una finalidad, en cambio toda proposición curricular da respuesta a las impresiones de determinadas comunidades y personas con perspectivas de mundo determinadas. Esto tiene una significación específica y es que los planes de la educación superior universitaria tienen una intención abiertamente establecida; es decir que deben estar esbozados desde un para qué: "¿Para qué preparamos a los profesionales? ¿Quién o quiénes se benefician con dicha formación? ¿Qué se impacta con dicha formación?" (Bolaños et al., 1999, p. 186).

Ahora, esto visto de una manera imparcial frente a los procedimientos del diseño curricular que se hayan interpretado y las categorías de intervención que hayan tenido en ellas los diferentes componentes sociales interesados, se obtiene como resultado la puesta en marcha del currículo, ejecutado por docentes a partir de actitudes epistemológicas individuales en un ámbito de heterogéneos componentes: el plan de estudios del cual se forma parte, los contenidos que se enseñan, el papel del docente (y de manera individual, la docencia en la educación superior universitaria) y el propósito de la educación superior en sí.

Con lo anterior se puede observar que la casualidad exclusiva de cada profesor -sea este consciente o no, y evidente o no-, igualmente hace parte de los procedimientos de la educación de los futuros profesionales. De tal manera que, a partir de las dos premisas anteriores es posible establecer que la educación superior universitaria tiene varios objetivos según los diferentes representantes adherentes a ella y de las posiciones epistemológicas que poseen, lo anterior sin determinar si poseen o no una expresión clara respecto a ellas: "el plan de estudios prescrito por una institución acota el trabajo docente pero, simultáneamente, es traducido y resignificado en la práctica por los profesores que poseen identidades disciplinarias o institucionales específicas" (Cardaci, 2005, p. 137). 
Por lo tanto, se puede afirmar que uno de estos enfoques que orientan el desempeño laboral universitario, la actuación profesional y la existencia en general, es precisamente el enfoque de género. De tal manera que una interpretación que se puede retomar en este argumento es la de representación de género, la cual es concebida como una mirada individual para percibir la vida en sociedad.

A partir de la idea de que ser hombre o ser mujer es un dato cultural y no biológico, y que la forma que adoptan las desigualdades sociales basadas en el sexo de las personas se relaciona con la manera en que se construye la oposición hombre/mujer en el imaginario social. (Palomar, 2005, p. 18)

Estas cogniciones estructurales de desigualdad están afectando de forma negativa al género femenino, tanto en su experiencia individual como académica y en la simbología colectiva estándar asociada a las mujeres y a los hombres que atraviesan de manera transversal la responsabilidad académica.

A nivel legal en Colombia, las medidas de sensibilización y prevención expuestas en el capítulo IV, artículo 11, (medidas educativas), enunciadas en la Ley 1257 de 2008 (diciembre 4), Diario oficial n. 47.193 del 4 de diciembre del 2008 plantean las siguientes funciones:

1. Velar porque las instituciones educativas incorporen la formación en el respeto de los derechos, libertades, autonomía e igualdad entre hombres y mujeres como parte de la cátedra en Derechos Humanos.

2. Desarrollar políticas y programas que contribuyan a sensibilizar, capacitar y entrenar a la comunidad educativa, especialmente docentes, estudiantes y padres de familia, en el tema de la violencia contra las mujeres.

3. Diseñar e implementar medidas de prevención y protección frente a la desescolarización de las mujeres víctimas de cualquier forma de violencia.

4. Promover la participación de las mujeres en los programas de habilitación ocupacional y formación profesional no tradicionales para ellas, especialmente en las ciencias básicas y las ciencias aplicadas.

Igualmente, el Ministerio de Educación Nacional lidera hoy una revolución educativa que amplía las ofertas y oportunidades a todos los colombianos durante su ciclo vital. Una transformación que entiende que la educación empieza desde la gestación y que lo que pasa durante los primeros cinco años de vida incide en el desarrollo integral del ser humano, estimula así el fortalecimiento del pensamiento crítico como un elemento clave para la formación de individuos útiles para la sociedad, una premisa irrefutable en el ámbito académico (Unesco, 1998).

En México, según lo planteado por el Instituto Nacional de las Mujeres en los contenidos del Programa Nacional de Igualdad de Oportunidades y No Discriminación contra las Mujeres (Proequidad) del Plan Nacional de Desarrollo 2000-2006, establece como uno de sus objetivos específicos, fomentar en todos los espacios sociales una educación para la vida que promueva el respeto por la diversidad, la tolerancia y el respeto a las diferencias de género de las personas, así como garantizar con igualdad y equidad en todos los niveles, tipos y modalidades educativas, atención específica a las niñas y mujeres para ampliar su participación en todos los campos de la actividad humana, con un sentido de justicia, al margen de prejuicios y discriminaciones. Y es así como los contenidos transversales del currículo se conciben como:

[...] contenidos culturales relevantes y valiosos, necesarios para la vida y la convivencia, que configuran de manera especial el modelo de ciudadano que demanda cada sociedad, a través de una educación en valores que permita a los estudiantes sensibilizarse y posicionarse ante los problemas, enjuiciarlos críticamente y actuar con un compromiso libremente asumido. (Martínez, 1995, p. 12)

Años atrás, la cuestión de la educación caracterizada por géneros se convirtió en un propósito 
de una exaltada polémica en los Estados Unidos entre la gente de la academia y la política, que en este propósito no persiguen las direcciones consabidas de sus perspectivas, tanto conservadoras como liberales, como allí acostumbran a llamarse.

De allí que unos se resisten a la enseñanza diferenciada examinando la igualdad "formal" o procedimiento igual y argumentan que el currículo solo para niños o niñas viola el principio de que a los individuos, en una situación análoga, se las debería tratar de manera equivalente. Al otro lado de la orilla, sus antagonistas aseveran que lo más relevante no es la igualdad "formal" sino la "sustancial". Se busca emparejar los resultados docentes que han traído como consecuencia el ser de menor relevancia para las niñas y los estudiantes pertenecientes a las minorías étnicas. Pretender apartar la dimensión de las potenciales discrepancias entre los géneros y su génisis implica hacer una labor deprimente. El inconveniente que se presenta está en concentrarse de forma única en la productividad o en las deducciones de las pruebas de aptitud; una orientación más prometedora para los docentes es analizar las divergencias entre los géneros a medida que se desenvuelvan, sin dejar de lado que hay diferencias dentro de cada conglomerado.

Resulta a la sazón el interrogante sobre cómo salvaguardar la incompatibilidad en el rendimiento de los dos géneros en el currículo escolar como en el de las aptitudes físicas "y es aquí donde se escenifica y se ponen en juego las políticas tanto educativas como la práctica educativa" (Salomone, 2007, p. 443).

Pérez, V. y Santamaría, C. (2015) afirman que en España, la llegada de la Ley Orgánica General del Sistema Educativo (Logse) en 1990 supone el primer momento en que se plantean modelos de igualdad y coeducación en la legislación educativa española. Sin embargo, la hegemonía de una masculinidad heroica, basada en la competitividad, la agresividad y el individualismo, pervive en gran medida en la cultura profesional y en la formación del profesorado de Educación Física (p. 1). La Ley General de Educación Básica de 1970 de este país, estableció la obligatoriedad de la enseñanza hasta los 14 años y es la primera ley educativa que planteó un único currículo para niños y niñas. Esta primera iniciativa en pos de la igualdad de género se inspiró en un modelo de asimilación: se generalizó un modelo educativo pensado para el género masculino al que se incorporaron las estudiantes, dejando de lado y quitando por tanto, validez a lo que había sido el modelo de educación femenina. Las estudiantes se debieron incorporar a un tipo de enseñanza pensada por y para los hombres (MEN, 2004).

Se destacó en España que la ministra de Sanidad, Servicios Sociales e Igualdad entre 2016 y 2018, Dolors Montserrat, anunciara que pretendía que en cada colegio hubiera un profesor que promoviera medidas "contra el machismo" y "la violencia de género". Esta figura de "coeducador", se dedicaría a la difusión de iniciativas que "promovieran la igualdad". También pretendía incluir en las etapas de educación secundaria y bachillerato, contenidos sobre el movimiento feminista para favorecer la igualdad entre hombres y mujeres en su trato para lograr la no discriminación y la prevención de la violencia de género.

Finalmente, se considera que cada una de las acciones que movilicen recursos hacia el currículo educativo y desarrollo del ser enmarcan posibilidades de existencia que conllevan personalidades saludables con dignidad humana para los que educar es preparar al hombre para la vida.

\section{Conclusiones}

Ya para finalizar vale la pena aseverar que es factible desarrollar un análisis comparativo entre el vínculo género-educación superior que inicie en los aspectos concernientes a las proposiciones o teorías e investigaciones actuales. La resignificación de las mujeres dentro del ámbito de la educación 
superior universitaria es una circunstancia que restituye, no es solo una metamorfosis sociocultural sino estructural; para lograrlo es inevitable dilucidar tanto habilidades como acciones unidas entre los múltiples representantes de la comunidad, las que conducirán a exhortar experiencias de igualdad y equidad entre los géneros dentro del contexto de la educación superior universitaria.

Para finalizar este análisis reflexivo sobre el currículo, se enfatizan las siguientes proposiciones y consideraciones:

- Potenciar desde el currículo educativo cambios reales que desafíen las concepciones tradicionalistas de género en la formación del profesorado.

- Dentro de todo proceso educativo la persona es un sujeto activo y creativo, esto permite, a través de un diálogo entre los actores involucrados, congregar la teoría curricular y el ejercicio pedagógico.

- Educar en ambientes positivos acompañados de valores de ciudadanía, tolerancia, respeto al otro, respeto a sí mismo, a la naturaleza, a la diferencia, conlleva una formación para la vida.

- Es urgente trabajar sobre métodos que se vivencian en el aprendizaje de habilidades y destrezas, según lo esperado y lo permitido para cada uno de los sexos, relacionándolos con las expectativas de éxito/fracaso y con el reconocimiento y valoración del esfuerzo personal y profesional.

- Fundamentar las concepciones sobre currículo, estas no solo deben permear la transmisión de conocimientos, sino trabajar de forma activa en la formación de personas con responsabilidad social que fortalezcan la calidad de vida.

- La Educación Superior, en sus funciones sustantivas, debe desarrollar estrategias transformacionales en el espectro educativo, en pos de los procesos curriculares tendientes al mejoramiento de sus estándares.

- La formación profesional debe dar cuenta de las variaciones y cambios potenciales en los contextos socioculturales.

\section{Referencias}

Bolaños, C., Vargas, A. y Velázquez, L. (1999). Hacia un diseño curricular alternativo en la educación superior. Educación, 23(especial), 185-194.

Cardaci, D. (2005). ¿Ausentes o invisibles? contenidos sobre las mujeres y los géneros en el currículo de licenciatura de universidades mexicanas. La ventana, $21,107-142$

Carreras, Ll., Eijo, P., Estany, A., Gómez, M., Guich, R., Mir, V., Planas, T. y Serrats, M. (1996). Los valores en el diseño curricular. En Carreras, Ll., Eijo, P., Estany, A., Gómez, M., Guich, R., Mir, V., Planas, T. y Serrats, M. Cómo educar en valores. Materiales, textos, recursos y técnicas (pp. 25-40) Nacea S.A. de educiones.

Casarini, M. (2010). Teoría y Diseño Curricular. Trillas.

Congreso de la República (2008). Ley 1257 de 2008. Por la cual se dictan normas de sensibilización, prevención y sanción de formas de violencia y discriminación contra las mujeres, se reforman los Códigos Penal, de Procedimiento Penal, la Ley 294 de 1996 y se dictan otras disposiciones. Diario Oficial, (47.193). https:// www.oas.org/dil/esp/LEY_1257_DE_2008_ Colombia.pdf

Díaz Barriga, A. (2006). La educación en valores: Avatares del currículum formal, oculto y los temas transversales. Revista Electrónica de Investigación Educativa, 8 (1).

De la Herrán, A. (2012). Currículo y pedagogías renovadoras en la edad antigua. Revista Iberoamericana sobre Calidad, Eficacia y Cambio en Educación, 10(4), 728623334. https://revistas.uam.es/index.php/reice/ article/view/3036

Dewey, J. (1952). Democracia y educación. Una introducción a la filosofía de la educación. Lozada. (Trabajo original publicado en 1859).

Díaz, Á. (2006). La educación en valores: Avatares del currículum formal, oculto y los temas transversales. Revista Electrónica de Investigación Educativa, 8, (1), 1-15. 
Durkheim, E. (1976). La educación como socialización. Sígueme.

Gallego, V. (2012). El currículo; concepciones, fundamentos e historia de su desarrollo en Colombia (Monografía de Licenciatura en Educación Preescolar). Universidad Santiago de Cali. https://www. monografias.com/docs113/curriculo-concepcionesfundamentos-e-historia-su-desarrollo-colombia/ curriculo-concepciones-fundamentos-e-historia-sudesarrollo-colombia.shtml\#bibliograa

La Francesco, V. y Giovanni, M. (2003). Propuesta de un nuevo concepto de currículo. En Giovanni, M. y La Francesco, V. Nuevos fundamentos para la transformación curricular a propósito de los estándares. Magisterio.

López, G. y Luna, A. (2011). El currículo: concepciones, enfoques y diseño. Revista Unimar, (58), 65-76. http://editorial.umariana.edu.co/revistas/index.php/ unimar/article/download/217/193

Magendzo, A. (2008). La escuela y los derechos humanos. Cal y Arena.

Martínez, M. J. (1995). Los temas transversales. Magisterio del Río de la Plata.

Ministerio de Educación Nacional (MEN). (2004). Desarrollo infantil y competencias en la Primera Infancia. Revolución Educativa Colombia aprende, MEN. https://www. mineducacion.gov.co/primerainfancia/1739/
articles-178053_archivo_PDF_libro_ desarrolloinfantil.pdf

Organización de las Naciones Unidas para la Educación, la Ciencia y la Cultura (Unesco). (1998). Declaración Mundial sobre la Educación Superior en el siglo XXI: Visión y Acción. París: Unesco

Organización de las Naciones Unidas para la Educación, la Ciencia y la Cultura (Unesco). (2016). Desglosar el objetivo de desarrollo sostenible 4 educación 2030, guía. Unesco.

Palomar, C. (2005). La política de género en la educación superior. La ventana, 21, 7-43.

Pérez, G. (2008). La educación como respuesta a los retos que plantea la escuela. Bordón. Revista de Pedagogía, 60 (4), 15-29.

Pérez, V., y Santamaría, C. (2015). Educación, currículum y masculinidad en España. Universidad de Alcalá. https://www.emakunde.euskadi.eus/contenidos/ informacion/gizonduz_dokumentuak/eu_def/ adjuntos/Educacion,\%20curriculum\%20y\%20 masculinidad\%20en\%20Espana.pdf

Sacristán, J., y Pérez, A. (1985). La enseñanza: su teoría y su práctica. Editorial Akal.

Salomone, R. (2007). Igualdad y diferencia. La cuestión de la equidad de género en la educación. Revista española de pedagogía, 238, 433-446. 